\title{
Autant en emporte le vent... Espaces et populations dans la métropole de troisième génération
}

Spaces and populations in the third generation metropolis

\section{Guido Martinotti}

\section{OpenEdition}

\section{Journals}

Édition électronique

URL : http://journals.openedition.org/belgeo/11736

DOI : $10.4000 /$ belgeo.11736

ISSN : 2294-9135

Éditeur :

National Committee of Geography of Belgium, Société Royale Belge de Géographie

Édition imprimée

Date de publication : 31 décembre 2006

Pagination : 407-418

ISSN : 1377-2368

\section{Référence électronique}

Guido Martinotti, « Autant en emporte le vent... Espaces et populations dans la métropole de troisième génération », Belgeo [En ligne], 4 | 2006, mis en ligne le 31 octobre 2013, consulté le 30 avril 2019. URL : http://journals.openedition.org/belgeo/11736; DOI : 10.4000/belgeo.11736

Ce document a été généré automatiquement le 30 avril 2019.

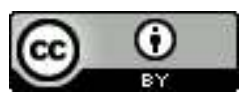

Belgeo est mis à disposition selon les termes de la licence Creative Commons Attribution 4.0 International. 


\title{
Autant en emporte le vent... Espaces et populations dans la métropole de troisième génération
}

\author{
Spaces and populations in the third generation metropolis
}

\author{
Guido Martinotti
}

\section{Introduction}

1 Depuis le début de la révolution industrielle, la population urbaine a cru selon la règle «plus le centre est grand, plus vite l'agglomération s'agrandit ». Mais, après 1971, les exceptions se sont multipliées. Entre les recensements de 1970 et de 1980, les USA ont vu la population non-métropolitaine croître plus rapidement que la population urbaine. Des tendances analogues ont été enregistrées dans de nombreux pays européens, bien qu'à différents degrés et en vagues successives. Ces résultats furent transmis au public avec insistance par les médias et par des scientifiques, comme une sorte de retrait du phénomène urbain, non sans un certain enthousiasme devant la découverte d'un tel revirement de l'histoire. Ainsi, dans ce dernier quart du XX ${ }^{\text {ème }}$ siècle, pendant la période la plus explosive de la transformation métropolitaine où la mobilité généralisée s'intégrait à la nature même de la nouvelle configuration urbaine, l'opinion publique fut induite en erreur et des élites politiques nostalgiques ont rêvé d'une improbable mort de la ville.

2 A. Giddens rappelle que la ville est une de ces formes sociales qui manifestent « une continuité trompeuse avec les ordres sociaux préexistants (A specious continuity with preexisting social orders)»(1990, p.6). Et B. McKibben qu'« une prédiction basée sur d'anciennes données peut être fiable s'il est raisonnable de supposer que le passé et l'avenir appartiennent au même univers statistique» (1998, p. 3). Il est difficile de supposer que les événements actuels appartiennent au même univers statistique que ceux qui se sont produits pendant l'expansion capitaliste des Trente glorieuses. Par suite, 
McKibben introduit l'idée de Terre2 (1998, p. 62), une planète radicalement différente de la précédente. Partant du concept des cycles d'accumulation systémique de Braudel, Arrighi propose une idée semblable (1994). Même si toutes les générations du XX ${ }^{\text {ème }}$ siècle ont pu penser vivre un changement historique grandiose, il n'est pas douteux que nous soyons aujourd'hui à la fin d'un cycle d'expansion et en pleine période de turbulence.

Or, bien qu'elle ait produit le corpus le plus substantiel de la connaissance empirique sur les établissements humains, l'écologie sociale reste basé sur une analyse de la compétition des différents groupes humains pour l'espace vital, où la fonction résidentielle est prépondérante. La grande majorité des statistiques urbaines reposent donc sur des modèles résidentiels et des unités résidentielles d'observation. Elles projettent des modèles statutaires et administratifs sur le territoire, qui interfèrent de plus en plus avec les changements que les statistiques officielles sont censées surveiller. En effet, à l'évidence, les nouvelles morphologies urbaines résultent en grande partie de la différenciation progressive de plusieurs populations gravitant autour des centres métropolitains et de la mobilité croissante de ces populations, que ce soit en nombre de personnes mobiles, de destinations atteintes, de distances parcourues ou de déplacements par jour. Les rapports entre population et territoire deviennent extrêmement dynamiques, et les concepts d'écologie sociale visant à la reconstruction de structures d'arrangement spatial sont obsolètes. La même critique peut être adressée aux analyses basées sur les classes. Des acteurs tels que les mouvements sociaux sont devenus de plus en plus visibles sur la scène urbaine. Les changements de la structure économique ont profondément affecté les modèles de classes établis dans les économies avancées, mais aussi dans d'autres pays. Et la croissante «fluidité » des processus sociaux redonne de l'importance à des concepts analytiques plus simples comme celui de populations. Aussi, les modèles fondés sur la continuité des concepts d'organisation municipale et se référant aux formes urbaines traditionnelles ne permettent plus, à mon avis, de décrire, et encore moins d'expliquer, comment la forme d'urbanisation contemporaine a changé l'expérience et les usages urbains. Sans chercher forcément à établir une théorie complète de tous ces changements, il faudrait au moins mettre à plat l'ensemble des concepts relatifs au phénomène urbain et définir des instruments analytiques utiles pour comprendre certains des processus qui créent la vie civique dans les métropoles contemporaines.

Trois volets composent cet article. Je commencerai par identifier les deux principaux processus sociaux relatifs aux développements technologiques touchant profondément les rapports personnels dans le temps et dans l'espace. Je proposerai ensuite une

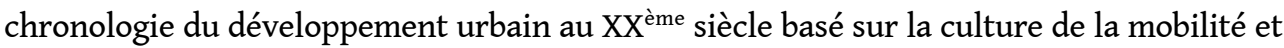
terminerai par quelques considérations à valoir pour la théorie sociologique des villes, dérivée d'un schéma heuristique pour décrire la morphologie sociale des cités contemporaines.

\section{Deux trajectoires technologiques}

5 Avec une perspicacité étonnante, H.G. Wells écrit à l'aube du XXème siècle :

«Les villes géantes [...], générées par des chemins de fer [sont] destinées selon toutes les probabilités à un tel processus de dissection et de diffusion qu'elles se réduiront presque à l'anéantissement [...] dans un futur mesurable en nombre d'années. Ces futures villes [...] représenteront une nouvelle phase complètement différente de la distribution humaine [...]. La ville s'étendra, englobant des zones considérables ainsi que de nombreuses caractéristiques de ce qui est la campagne à 
l'heure actuelle [...]. La campagne absorbera bien des caractéristiques de la ville. La

vieille antithèse [...] cessera, les limites disparaittront complètement » (Wells, 1902). de l'industrie manufacturière à celle des services, à cause de décisions lourdes d'aménagement du territoire mais aussi d'un enrôlement général dans le projet d'une ville à grande mobilité. Le système de mobilité, basé d'abord essentiellement sur le transport des produits bruts ou manufacturés, et sur le mouvement des capitaux, a intégré les déplacements continuels et récursifs des individus. Ce processus n'aurait pas pu avoir lieu sans un passage culturel long et envahissant, de l'intégration spatiale traditionnelle des sociétés préindustrielles à l'évaluation positive de la mobilité dans nos sociétés. Dans les sociétés traditionnelles, les voyages étaient difficiles, dangereux et coûteux; ils étaient vus comme des activités inconvenantes. A notre époque, la mobilité est considérée comme un élément nécessaire et positif de la vie sociale, est très estimée sous toutes ses formes. La mobilité est devenue une valeur en soi et la vitesse, toujours croissante, un objectif à atteindre à tout prix, soutenant un macro-système technologique coûteux, aux fins surtout militaires mais également utilisé par les réseaux de transports civils. Le voyage fait maintenant partie des divertissements (Going there is half of the fun).

Pour comprendre comment la configuration urbaine a acquis sa forme actuelle et comment celle-ci touche le discours public, il est donc important de suivre deux processus à la fois entremêlés et indépendants : la généralisation de la mobilité des masses et la diffusion des instruments permettant l'échange rapide d'une grande quantité d'informations, ou technologie de l'information et de la communication (TIC). Comme le montre A. Gras (Gras, 1993, 2003), un développement technologique ne doit pas être considéré isolément mais au sein d'un "macro-système", intégrant l'effort technologique spécifique dans son contexte économique, normatif, social et culturel. Un cas classique est la diffusion de l'électricité, qui nécessite un macro-système complexe allant des barrages aux ampoules. Une fois que certaines bifurcations ont été éclaircies, une « trajectoire technologique » émerge des évolutions de la filière.

Or, deux trajectoires technologiques spécifiques ont interagi avec la morphologie sociale de la configuration métropolitaine, c'est-à-dire celle de la mobilité - à grande énergie - et celle de l'information - à énergie (relativement) basse. Des décisions prises par des acteurs importants, industriels, bâtisseurs, urbanistes, ont eu des effets visibles, mais on a rarement bien compris que la combinaison de décisions multiples et diffusées des acteurs de moindre importance pouvait aussi avoir des effets à une grande échelle.

\section{Appareils ménagers : temps, performance et espace}

9 Pour comprendre les rapports étroits entre la voiture et la ville, nous devons observer aussi les changements survenus dans les maisons, en particulier ceux qui concernent la trajectoire technologique de l'information. L'économie du transport nous informe que le temps et les coûts des déplacements sont partiellement substituables avec les valeurs foncières urbaines. Or les valeurs foncières reflètent les nécessités de la vie et de l'habitat, qui ont un rapport étroit avec l'information.

10 La miniaturisation a été la trajectoire technologique des supports d'informations, permettant leur diffusion dans un nombre croissant de dispositifs concernant notre vie quotidienne. L'impact de ce processus sur l'organisation et la localisation de la 
production, sur l'usine et la ville industrielle est bien connu. Il ne doit pas faire oublier le bouleversement des dispositifs de traitement des informations dans nos demeures. S'il y a des machines dans les usines, il y en a aussi, depuis longtemps, dans les habitations (anciennes machines comme les metates ou autres broyeuses à céréales, lampes pour l'éclairage, échelles pour entrer et sortir, cheminées et fours pour le chauffage et la cuisine, etc.).

11 L'époque moderne a vu croître le nombre d'objets, grâce notamment, comme l'avait remarqué S. Gideon, dans son oeuvre classique, à la miniaturisation des sources de chaleur (1954). Mais il faut distinguer deux familles d'objets domestiques. Les objets de la première famille sont, comme les machines des usines des time and labour saving devices (dispositifs économisant le temps et la main-d'oeuvre). Ainsi, le poêle en fonte à charbon ou à bois a eu un impact extraordinaire sur la vie des gens ordinaires, en particulier des femmes, car il économisait à la fois du temps et de l'espace. Il aurait ainsi favorisé le mouvement des suffragettes à qui il faisait gagner du temps. Le nombre des time and labour saving devices a augmenté continuellement jusqu'à la situation actuelle où nombre de fonctions ménagères sont désormais effectuées par des dispositifs mécaniques ou électriques. Le marché de ces objets arrive aujourd'hui à saturation car le temps économisé au cours d'une journée est fixe. Le prochain bond vers la domotique ou l'habitation robotisée, tant vantée, paraît plutôt lointain. Mais le total du temps économisé est devenu l'objectif d'une nouvelle génération d'appareils domestiques: les time consuming devices (appareils absorbant du temps) dont le plus vorace est, bien sûr, le téléviseur qui prend plusieurs heures par jour à de grandes masses de population. De la radio au téléphone, de la hi-fi aux caméras, webcams et, bien entendu et plus récemment, à l'ordinateur individuel sous toutes ses formes et associations en évolution rapide, les time consuming devices pénètrent dans les maisons. Ce nouveau marché n'est pas facile à saturer car ces objets n'ont pas à être utilisés d'une façon exclusive et synchrone ; cela se voit bien dans nos placards et nos greniers.

Un autre processus avance. Dans les établissements traditionnels, on se rendait au puits du village pour chercher l'eau, c'était aussi une occasion pour bavarder, échanger des informations et vaquer à d'autres activités publiques. Aujourd'hui les canalisations permettent d'avoir de l'eau directement chez soi en ouvrant un robinet, ce qui économise beaucoup de temps et de fatigue mais supprime des contacts sociaux (cf. Mitchell, 1995). Les rapports sociaux n'ont pas disparu mais le lien entre l'habitant et la source d'eau a été remplacé par une technostructure «légale et rationnelle " contribuant à une division minutieuse du travail dans la société. La même chose s'est produite avec l'information. Dans la ville traditionnelle, la piazza était le lieu de rencontre servant à échanger produits et informations. Si quelqu'un du palazzo avait une communication à faire, il envoyait un héraut à la piazza diffuser les nouvelles. Aujourd'hui il suffit de tourner le bouton de n'importe lequel des nombreux appareils donnant des informations pour recevoir, dans son petit salon, le monde entier et l'idéologie des opérateurs de médias. Car la télévision n'est pas un regard sur le monde mais un regard du monde sur nous, un regard très puissant, à travers lequel «la rue entre dans la maison" comme l'avait imaginée Boccioni en 1914 dans son brillant tableau du même nom. Comme dans la substitution du puits par le robinet, les appareils absorbant le temps remplacent le contact direct par une technostructure très complexe, à travers laquelle l'agora est lentement aspirée dans l'habitation, créant des transformations profondes dans la structure sociale, le processus politique, et les discours publics en général. 


\section{Trois phases dans la mobilité urbaine} ville. Avec des appareils économisant temps et travail, et d'autres utilisant ce temps sous le signe du "divertissement ", la maison devient de plus en plus accueillante. Nous y passons de plus en plus de temps : rien que pour la télévision, une moyenne de quelque $3 \mathrm{~h} 1 / 2$ par jour (4h en Italie). Ces appareils demandent davantage d'espace - pensons au nombre de prises de courant dans une pièce contemporaine. Avoir des foyers plus confortables, passer plus de temps chez soi, y mettre des appareils partout - le reste : revenu, taille de la famille, style de vie, etc. étant inchangé - pousse à chercher des appartements plus spacieux. Etant donné la structure de l'occupation du sol et des valeurs foncières, ceteris paribus, il s'agit donc de chercher une demeure en périphérie, pour autant que l'économie sur les frais immobiliers compense le coût des voyages. Alors le temps passé en voiture particulière s'allonge. Celle-ci doit être confortable et, si possible, informatisée. Par consequence, les voitures consomment toujours plus d'énergie, polluent et détruisent l'environnement. Les coûts énergétiques sont énormes mais les coûts sociaux ne le sont pas moins. La périurbanisation (ou sprawl) détruit l'« urbanité » des centres-villes désertés et la participation civique. Cela s'est largement produit dans les villes nord-américaines dépendantes de la voiture. A l'heure actuelle, la mobilité est devenue le plus grand atout et l'un des problèmes les plus urgents de la vie urbaine contemporaine. Ce changement global s'est étalé sur la plus grande partie du XX⿳亠े⿵冂丶 ${ }^{2}$ siècle et, avec le recul, nous pouvons identifier dans ce développement trois phases, que, suivant les suggestions de A. Gras, j'identifie aussi par des critères économiques et culturels.

La première phase, la ville $T$, va de la fin des années 1920 à 1945 ; elle a créé les métropoles de première génération (Martinotti ; 1992), en particulier aux USA. Son essor et venu du Fordisme, qui fut si envahissant qu'A. Huxley fit de Ford, dans son célèbre Brave New World (1932), une divinité adorée sous le signe T par une société massifiée empreinte du doux credo du bonheur collectif. La plupart des normes et connaissances sociales au sujet de la voiture furent produites à cette période. Parmi d'autres courants culturels, les Futuristes Italiens contribuèrent à la diffusion de l'idée de la valeur de la mobilisation de la société. Et l'idée circula. Miasto, masa, machina (ville, masse, machine) est la représentation polonaise de la métropole industrielle rendue immortelle par l'enchevêtrement de Charlie Chaplin avec l'usine fordiste. La crise de 1929 et la guerre mirent des obstacles à ce développement.

La deuxième phase est celle de la Car Happy City ou ville bagnole. Commencée après la guerre, elle s'est déroulée pendant l'apogée du développement capitaliste des Trente glorieuses, en Occident et aussi dans des pays en développement. Elle vit le triomphe de la société de l'automobile et du modèle fordiste. Los Angeles et Detroit émergèrent comme prototype des établissements humains urbains de vaste échelle, caractérisés par la possession généralisée de la voiture particulière. L'automobile devint un instrument de libération extraordinaire, pour les enfants échappant au contrôle sévère de leurs familles (cf. James Dean dans East of Eden, 1955), pour les classes moyennes évitant le conflit et l'influence néfaste des quartiers déshérités du centre-ville, pour les masses de travailleurs se libérant de l'oppression des zones industrielles et des habitats collectifs, tous pratiquant en masse le rite migratoire du week-end et des vacances d'été. Le mot d'ordre 
était « en route ». La Road 66, chantée par Nat King Cole, l'Autostrada del Sole en Italie ou la Nationale 7, vantée par Charles Trénet pour « Chasse[r] les aigreurs et les acidités/Qui font l'malheur des grandes cités ", symbolisèrent l'ouverture de nouvelles frontières. On peut dire, pour utiliser un concept plus technique, que les citoyens de cette phase ont épousé le « projet automobile ». Ils ont souscrit à ce projet (Molotch, 2003).

La troisième phase, dans laquelle nous nous trouvons, est celle de la ville environnementaliste, et c'est un moment de crise urbaine profonde qui correspond au crépuscule $\mathrm{du}$ fordisme et à la diffusion d'une post-fordisation du territoire. Les communautés péri-urbanisées commencent à payer les coûts collectifs de la croissance précédente, de la crise du pétrole et des restructurations successives de l'industrie manufacturière. En même temps, comme l'a montré A. Bagnasco, dans son étude sur Turin (1986, pp. 22-23), les règles de l'organisation fordiste viennent se mêler aux suggestions du political exchange favorisées par la diffusion des medias et accompagnant les incertitudes de ce système de régulation. Face à ces incertitudes, elles se replient sur elles-mêmes, par exemple dans le «syndrome NIMBY ». Cependant il faut reconnaître que dans les MDR (More Developed Regions), notamment en Europe où l'énergie et les considérations environnementales ont été plus rigoureuses, les experts insistent plus sur les problèmes de qualité et de conservation que sur les inquiétudes précédentes. Il est sans doute trop optimiste de qualifier l'urbanisation actuelle d'environnementaliste car le gaspillage d'énergie, la pollution et la congestion y sont énormes et ne diminuent pas. Toutefois il est indéniable que, depuis la "ville bagnole », un changement d'orientation remarquable a été pris et le problème mis à l'ordre du jour. Même par G.W. Bush (discours du 20 février 2006). En même temps les villes des gigantesques MURs (Mega Urban Regions), surtout du sud-est asiatique semblent être en train de répéter les phases précédentes de l'urbanisation du XX⿳亠丷⿵冂丶 ${ }^{m e}$ siecle.

\section{Morphologie sociale des générations métropolitaines}

Ces changements ont aussi touché la morphologie sociale des villes, différenciant les diverses générations de métropoles. Pour le comprendre, nous devons nous échapper du modèle conventionnel de la pensée écologique strictement sociale, de l'analyse des classes, et revenir au concept simple, mais pas tout à fait banal, de population, à savoir un groupe d'individus définis par un ou plusieurs traits communs élémentaires. Ce concept a été, à mon avis, trop souvent réduit à l'application mécanique de la fonction résidentielle, dont j'ai dit plus haut combien son importance avait été exagérée par nos outils conceptuels. Mais les populations peuvent être des acteurs puissants dans la transformation des villes sans qu'il soit nécessaire de faire référence à des assomptions théoriques sur la rationalité collective de leur comportement, comme par exemple pour les classe sociales. La nouvelle configuration de la morphologie urbaine est en grande partie le produit de la détermination progressive de plusieurs populations gravitant vers les centres métropolitains, en particulier de quatre populations, de plus en plus différenciées les unes des autres, et identifiables au moyen d'un schéma heuristique simple, mais efficace. Ce schéma est basé sur trois variables à modalité dichotomique (oui/non) : habiter, travailler et consommer. Bien entendu la notion de consommation n'a jamais été loin de l'idée de la ville, à partir de la fameuse définition de Max Weber. Toutefois les analyses dites écologiques, soit basées sur des données urbaines territorialement agrégées, sont fondées très largement sur des points d'observation liés à 
l'habitation, tels que les recensements ou les registres des populations comme l'anagrafe italienne. Seulement pour une partie mineure ces informations sont basées sur des données sur l'activité productive, comme dans les recensements économiques, qui portent en général plus sur les unités productives que sur les caractéristiques individuelles. A partir de cela, on peut dire que la grande partie des données statistiques officielles, sur lesquelles on fait les analyses d'écologie sociale, représentent des populations nocturnes, soit la ville endormie, et dans une bien moindre mesure la ville travailleuse, comme l'ont montré D. Pumain et F. Godard (1994). D'un autre côté, aucune statistique officielle ne décrit la ville active dans la consommation. Or cet état de choses ne nuisait pas sérieusement à l'analyse spatiale dans la ville traditionnelle, où les différentes fonctions se déroulaient dans un espace restreint et en superpositions, mais devient trompeur là où les fonctions sont très différenciées du point de vue territorial, voire dans la situation métropolitaine (métropole de première génération, dans mon schéma) avec un noyau central (core) et une vaste banlieue (periphery). Et elles deviennent encore moins capables de décrire la morphologie sociale des villes avec la diffusion massive des populations non résidentes dans la ville contemporaine. En définissant une population sur la base des modalités de trois variables on peut identifier quatre populations urbaines, habitants, pendulaires (commuters), usagers de la ville (city users) ou hommes d'affaires métropolitains (metropolitan business persons) et en évaluant la présence de chaque population on peut identifier types ou générations de métropoles. Il s'agit d'un schéma heuristique basé sur des variables élémentaires et analytiquement simple, qui a récemment été retenu par le rapport sur les villes du UN Habitat (2004, pp. 52-54).

\section{De la ville traditionnelle à l'aire métropolitaine. La métropole de première génération}

Dans la ville traditionnelle, sur laquelle la pensée actuelle sur la vie urbaine est encore en grande partie fondée, les habitants, à savoir la population vivant dans la ville, coïncidaient en grande partie avec la population travaillant dans cette ville. Pendant des millénaires, et jusqu'à des temps très récents, les limites de la ville englobaient ces deux populations dans une unité spatiale, entourée par des murs et nettement séparée du reste du territoire.

Le développement métropolitain précoce qui se produisit aux USA à partir des années 1920, et en Europe après la Seconde guerre mondiale, apparaît essentiellement comme une différenciation croissante de deux populations : les habitants et les travailleurs. La migration quotidienne, ou navette, est la conséquence de ce processus, et elle est l'expérience circadienne la plus caractéristique et la plus répandue chez l'habitant des villes du milieu du XX ${ }^{\text {ème }}$ siècle. Du point de vue sociologique, la structure des classes de la population des navetteurs est tout à fait différente aux USA et en Europe, et parfois même presque symétrique, mais la morphologie urbaine produite par cette différenciation est similaire. Le résultat est ce que j'appelle métropole de première génération (ou métropolisation précoce), dont la délimitation se base sur les Régions urbaines fonctionnelles (RUF), les Daily Urban Systems (DUS) ou les bassins de migration quotidienne, englobés dans le concept d'aire métropolitaine. 


\section{City users et métropole de seconde génération}

migrants quotidiens a largement coexisté avec les structures urbaines traditionnelles. Mais la plus grande mobilité des individus, associée à l'élévation générale des revenus et au développement des loisirs, a permis à une troisième population d'émerger : les city users ou utilisateurs des villes. Ce sont des personnes qui vont en ville pour utiliser ses services privés et publics : shopping, cinéma, musées, restaurants, services de santé et éducatifs. Cette population croissante agit fortement sur la structure des villes et utilise les lieux de façon assez incontrôlée. Certaines villes ont une population d'habitants réduite, une population de navetteurs légèrement supérieure, mais une population bien plus grande de city users. Venise est un cas extrême, mais le phénomène touche nombre de villes dans le monde, et pas seulement des villes d'art, ce qu'une étude du Financial Times du 25-26/1/2003 traduisait par ce titre : « Nous sommes tous devenus Vénitiens ». En effet, étant donné que leur économie dépend de plus en plus de ces populations nonrésidentes, la plupart des villes, quelle qu'en soit la dimension, désirent attirer leur part de city users. Contrairement aux navetteurs, les city users utilisent les lieux publics des villes, et en abusent parfois. En emportant les ruines du mur de Berlin comme souvenir, les city users ont réalisé à leur façon la célèbre prophétie de Marx et Engels, selon laquelle toutes les murailles de Chine seraient battues en brèche par « la grosse artillerie du bon marché des produits [du capitalisme, contraignant] à la capitulation les barbares les plus opiniâtrement hostiles aux étrangers » (Marx, Engels, Manifeste du parti communiste).

Cette population augmente mais elle est difficile à évaluer par les statistiques officielles, qui s'occupent toujours, d'abord, des habitants, un peu des navetteurs, et pratiquement pas des city users. Pourtant, la concurrence acharnée pour recevoir les Jeux Olympiques prouve bien l'importance croissante que les élites locales attachent à ces city users. En outre, ce phénomène est loin d'être limité aux villes touristiques ou aux pays occidentaux. La population des city users ne se limite pas non plus au temps libre ou au shopping. La métropole contemporaine compte des populations de passage, nomades ou, plus généralement, PNR ou populations non-résidentes (Nuvolati, 2002). Elle leur a créé des endroits tels que les grandes surfaces, les centres commerciaux, mais aussi les stades, aéroports, gares, etc., que les intellectuels n'aiment pas particulièrement. Le concept de «non-lieu » ou «non-place urban realm », introduit à l'origine par feu Melvin Webber, a été popularisé par Marc Augé. Il est a contrario révélateur d'une nostalgie commune pour des endroits fortement marqués par l'histoire et communiquant, en principe, une identité sociale finalement trompeuse. Le succès d'un concept théoriquement si faible serait incompréhensible autrement. En fait, si je peux me permettre un jeu de mots, les nonlieux sont des nos-lieux, c'est-à-dire ces marques collectives ou thèmes collectifs (Romano, 1993, 2005) que toute communauté urbaine a créés en son temps.

\section{La métropole de troisième génération}

Mais une quatrième population métropolitaine apparaît: celle, encore petite, très spécialisée mais en expansion, des metropolitan business persons ou hommes et femmes d'affaires métropolitains. Ce sont des gens qui se rendent dans les pôles urbains pour gérer leurs affaires et établir des contacts professionnels : hommes d'affaires et professions 
libérales en visite chez leurs clients ou en congrès, consultants et managers internationaux. Cette quatrième population se caractérise par une très grande disponibilité de fonds aussi bien privés que corporatifs. Elle ne reste habituellement que quelques jours, parfois plus, mais ce n'est pas une population permanente. Elle passe une partie de son temps à conclure des affaires, utilise la ville partiellement. C'est une population de citadins experts, connaissant les différentes parties du monde, très sélectifs quant aux habitudes du shopping, aux hôtels et restaurants, aux activités culturelles telles que les concerts, expositions, musées, mais aussi saunas et gymnases. Les affaires et le tourisme d'élite vont de pair. Cela touche la morphologie et les fonctions des grandes villes bien au-delà de l'importance numérique du groupe. Dans l'intérêt d'une classification complète, nous pouvons dire que cette métropole encore naissante est une métropole de troisième génération (ou tardive).

Les city users et les metropolitan business persons sont le produit de l'industrie des services. Un aspect sociologique peu exploré de cette industrie est le fait qu'elle nécessite beaucoup de déplacements de personnes alors que les industries manufacturières déplacent des produits. Il n'y a aucun doute que le tourisme, le tourisme urbain en particulier, est une force motrice puissante dans ce processus de changement, comme le démontrent Judd et Fainstein (1999). Mais les city users forment aussi, à mon avis, une grande partie de la population de consommateurs indispensables au système productif de la ville contemporaine (Sassen, 1991).

\section{Structure des classes dans la nouvelle métropole}

La métropole des city users et des metropolitan business persons n'est pas dépourvue des connotations de classe. Au contraire, on peut soupçonner que de nouvelles classes apparaissent. La quatrième population forme de plus en plus ce que j'appellerais une bourgeoisie transnationale qui ne vit pas dans une ville mais dans des villes ou, mieux encore, entre des villes (formule de feu Roy Drewett). Elle révèle l'internationalisation ou globalisation des pôles métropolitains et affecte la morphologie et les fonctions de tous les grands centres urbains. Le concept d'hyperbourgeoisie, introduit par D. Duclos a plusieurs points de contact avec ce que je pense : «L'hyperbourgeoisie conjugue positions de puissance et signes de cohésion culturelle. »(Duclos, 1998, p. 16).

Ce groupe social est encore assez varié mais il est de plus en plus identifiable (Sklar, 2001) : managers des entreprises multinationales privées et publiques, telles que le grand nombre des organisations internationales - ONU, BIT, UNESCO, OCDE, FAO, OMS ainsi que la famille croissante des ONG- Organisations non gouvernementales, hommes d'affaires, consultants internationaux, universitaires, acteurs, sportifs, etc. Cette population demande des services assez similaires partout dans le monde : hôtels, bureaux et lieux de réunion, restaurants, centres commerciaux, etc. Le résultat se voit déjà dans de vastes secteurs de plusieurs villes du monde.

\section{Maintenant mais pas ici : temps, espace et technologie dans la méta-cité}

On a tendance à considérer les développements des TIC séparément de la qualité de l'environnement produit par les systèmes métropolitains. C'est là une mauvaise 
représentation. L'interaction entre ces développements techniques, en particulier ceux qui sont relatifs à l'Internet, et les paysages métropolitains est plus subtile, largement inexplorée et, à bien des égards, plus dévastatrice que nous ne le croyons habituellement. L'évolution de la morphologie urbaine, la mobilité, la ségrégation, la qualité du logement... en bref l'évolution du monde physique et social de la ville, ne peut pas être séparée de celle de ce qu'on appelle la Société de la Connaissance. Cette considération est de plus en plus importante, compte tenu des problèmes posés par l'extension physique due à la création de MURs, Mega Urban Regions ou de la Mega-city (Hall, 2006) Comme nous l'avons vu plus haut la disponibilité de l'information chez soi se combine d'une façon synergique avec la disponibilité de la voiture individuelle qui permet de localiser ce " chez soi » assez librement dans un territoire étendu. Plus précisément à l'intérieur des aires dans lesquelles le coût de l'habitation tend à équilibrer le coût du déplacement. Il s'agit d'un processus en constant élargissement dans le cours du $\mathrm{XX}^{\text {ème }}$ siecle, qui a produit un progressif déplacement vers l'extérieur des limites des villes. Une sorte de récession des limites qui semble caractériser le monde contemporain dans plusieurs directions, physiques et cognitives. Mais l'élargissement des limites, et leur affaiblissement, comme l'avait montré S. Rokkan, récemment repris par M. Ferrera (2006, pp. 11-52) est très strictement lié à la force ou à la faiblesse des liens intérieurs du système social donné. Selon la version de A.Giddens, boundaries et boundedness sont strictement liés (1990, pp.11-12).D'où la nécessité d'employer divers points de repère dans l'analyse urbaine. Dans l'article nous avons suggéré de regarder surtout la mobilité et nous avons essayé d'esquisser différentes typologies des villes sur la base de styles de mobilité et de l'attitude envers la voiture individuelle. D'abord avec une adhésion complète et insouciante au projet automobile lié au fordisme, et ensuite avec une attention croissante pour les conséquences sociales et environnementales de la diffusion périurbaine et les types ou générations des formes métropolitaines.

Les problèmes sont devenus plus aigus à l'heure actuelle, alors que la ville est en train de changer profondément d'une façon qui parfois échappe à l'expérience quotidienne et aux idées acquises. C'est pourquoi, nous devons être extrêmement attentifs en utilisant des concepts et des mots pour décrire la ville sociologique contemporaine et établir le rapport entre ce que nous voyons et ce que nous imaginons ou désirons. L'image d'une vie urbaine largement dominée par la consommation et, en particulier, d'une ville peuplée non seulement par ses habitants, mais aussi par une quantité croissante de populations non résidentielles, voire des usagers de la ville, n'est pas uniquement un point de vue abstrait, mais une réalité qui se déroule chaque jour sous nos yeux et qui concerne une proportion croissante de l'économie urbaine et des soucis des administrateurs des villes dans le monde entier.

\section{BIBLIOGRAPHIE}

AMIN A. \& THRIFT N. (2002), Cities. Reimagining the urban, Cambridge, Polity. 
ARRIGHI G. (1994), The long twentieth century: money, power, and the origins of our times, Verso, London-New York.

AUGÉ M. (1992), Non-lieux Paris, Seuil.

BAGNASCO A. (1986), Torino. Un profilo sociologico, Turin, Einaudi

BEAUREGARD R.A. (1993), Voices of Decline, Cambridge Mass, Blackwell.

BERRY B.J.L. (éd.) (1976), Urbanization and counter-urbanization, Urban Affairs Annual Review 11, Beverly Hills, Sage publications.

BODY-GENDROT S., LE GUENNEC N. (1998), Mission sur les violences urbaines, Paris, La Documentation Française.

BRUNET R. (1989), Les villes européennes, Paris, Datar.

CATTAN N., PUMAIN D., ROZENBLAT C. et SAINT-JULIEN T. (1994), Le Système des Villes Européennes , Paris, Anthropos.

CASTELLS M. (1996), The Information Age: Economy, Society and Culture. vol. I, The Rise of the Network Society, Oxford, Blackwell.

CATTANEO C. (1972), La città come principio, Venise, Marsilio.

De MATTEIS G (éd.) (1992), Il fenomeno urbano in Italia, Milan, Angeli.

DUCLOS D. (1998), « La naissance de l'hyperbourgeoisie », Le Monde Diplomatique, Août, pp. 16-17.

FERRERA, M (2006), The Boundaries of Welfare, Oxford, Oxford University Press.

GARREAU J. (1988), Edge City. Life on the New Frontier, New York, Doubleday.

GIDDENS A. (1990), The Consequences of Modernity, Stanford, Stanford University Press.

GIDEON S. (1954), Spazio tempo architettura, Milan, Hoepli.

GRAS A. (1993), Grandeur et dépendance, PUF, Paris.

GRAS A. (2003), Fragilité de la puissance, Paris, Fayard.

HALL P. et PAIN K. (2006), The Polycentric Metropolis, Learning from Mega-city regions in Europe, Londres, Earthscan.

JUDD D. R. et FAINSTEIN S. (1999), The Tourist City, New Haven, Yale University Press.

LOGAN J. R. et MOLOTCH H. (1987), Urban Fortunes : Making Place in the City, Berkeley, University of California Press.

McKIBBEN W. «A particular moment in history », The Atlantic Monthly, Mai 1998, pp. 55-78

MARTINOTTI G. (1992), Metropoli. La nuova morfologia sociale della città, Bologne, Il Mulino.

MASSER I. et al. (1992), The Geography of Europe's Futures, Londres, Belhaven Press.

MITCHELL W. (1995), City of bits. Space, Place and the Infobahn, Cambridge, MIT Press.

MOLOTCH H. (2003), Where Stuff Comes From, New York, Routledge.

NUVOLATI G. (2002), Popolazioni in movimento, Bologne, Il Mulino.

PUMAIN D. et GODARD F. (1996), Données Urbaines, Paris, Anthropos.

ROMANO M. (1993), L'estetica della città europea. Forme e immagini, Turin, Einaudi.

ROMANO M. (2004), Costruire le città, Milan, Skira. 
SASSEN S. (1991), The Global City, New York, Londres, Tokyo, Princeton, Princeton University

Press.

SASSEN S. (2006), Territory, Authority, Rights, Princeton, Princeton U.P.

SAUNDERS P. (1986), Social Theory and the Urban Question, Londres, Hutchinson.

SERNINI M. (1996), Terre sconfinate. Città, limiti, localismo, Milan, Angeli.

SKLAR L (2001), The Transnational Capitalist Class, Londres, Blackwell.

SORKIN M. (éd.) (1994), Variations on a Theme Park. The New American City and the End of Public Space, New York, Noonday.

SUDJIC D. (1993), The 100 mile city, Londres, Flamingo.

UN Habitat (2004), The State of the World's Cities 2004-2005.Globalization and Urban Culture, Londres, Earthscan.

WEBBER M.M. (1964), Explorations into Urban Structure, University of Pennsylvania Press, Philadelphia (tr. fr. L'Urbain sans lieu ni bornes, préface et annotation de Françoise Choay, trad. franç., La Tour d'Aigues, les éditions de l'Aube, 1996).

WELLS H. G. (1902), Anticipations of the reaction of mechanical and scientific progress upon human life and thought, Londres et New York, Harper \& Bros.

\section{RÉSUMÉS}

Le présent essai vise à placer les changements de la morphologie physico-sociale des villes contemporaines dans un cadre théorique, en tentant de décrire comment la combinaison de deux trajectoires technologiques interconnectées, l'une dans le domaine de la mobilité, l'autre de l'information, affecte la forme physique de la structure urbaine actuelle qui encourage la création et la diffusion des MUR (Mega Urban Regions). Dans ce processus, un rôle déterminant est attribué au développement d'appareils ménagers, qui économisent temps et travail (réfrigérateurs, lave-linge), et d'autres qui consomment le temps épargné mais connectent aussi le foyer avec le monde : téléphone, TV, internet. Plus de temps chez soi signifie une pression en faveur de résidences plus spacieuses et, toutes autres choses étant égales, ces dernières se rencontrent plus facilement dans les zones périurbaines de la nouvelle métropole. Ce développement a été favorisé par les vagues successives de motorisation privée qui depuis peu font face au défi posé par les questions d'environnement et d'énergie. Actuellement, la métropole change aussi sa morphologie sociale, avec l'émergence des NRP (populations non résidentes).

This essay tries to put changes in physical and social morphology of contemporary cities in a theoretical frame by attempting to describe how the combination of two interrelated technological trajectories, one in the mobility and the other in the information field, affects the physical shape of contemporary urban structure promoting the creation and diffusion of MURs, Mega Urban Regions. In this process an important role is attributed to the development of machines for the house that save time and labour, such as the refrigerator or the washing machine, and of those that consume the time saved, but also connect the den with the outside world sucking the agora into the house, such as the telephone, TV, or Internet. More time at home means a pressure for roomier abodes and, other things being equal, the latter can be found more easily in the periurban areas of the new metropolis. This development has been favoured by successive waves of private motorisation that only recently are being challenged by 
environmental and energetic issues. Contemporarily the metropolis changes also its social morphology with the emergence of Non Resident Populations NRPs.

\section{INDEX}

Mots-clés : métropoles, MUR, meta-city, trajectoires technologiques, innovation,

communication, mobilité, technologies de l'information et de la communication, populations non résidentes

Keywords : metropolis, Mega Urban Region, Meta-city Region, technological trajectories, mobility, information and communication technologies, non resident populations

\section{AUTEUR}

\section{GUIDO MARTINOTTI}

Università degli Studi di Milano-Bicocca, guido.martinotti@unimib.it 